\title{
Permissible Value
}

National Cancer Institute

\section{Source}

National Cancer Institute. Permissible Value. NCI Thesaurus. Code C41109.

The exact names, codes and text that can be stored in a data field in an information

management system. 